\title{
Discours féministe et néo-malthusianisme : les effets pervers \\ d'une mésalliance
}

FEMINIST DISCOURSE AND NEO-MALTHUSIANISM: THE

ADVERSE EFFECTS OF A MISALLIANCE

DISCURSO FEMINISTA Y NEOMALTHUSIANISMO : EFECTOS PERVERSOS DE UNA UNION DESACERTADA

\section{Maria De Koninck}

Volume 27, numéro 2, automne 1998

Malthus

URI : https://id.erudit.org/iderudit/010251ar

DOI : https://doi.org/10.7202/010251ar

Aller au sommaire du numéro

Éditeur(s)

Association des démographes du Québec

ISSN

0380-1721 (imprimé)

1705-1495 (numérique)

Découvrir la revue

Citer cet article

De Koninck, M. (1998). Discours féministe et néo-malthusianisme : les effets pervers d'une mésalliance. Cahiers québécois de démographie, 27(2), 253-265. https://doi.org/10.7202/010251ar
Résumé de l'article

$\mathrm{Au}$ cours des dernières années, on a assisté à ce qui semble étre une alliance entre les tenantes du discours féministe et les néomalthusiens sur la question de la planification des naissances. Cette note présente une réflexion sur une association qui peut étre qualifiée de " mésalliance ", car les deux discours sont issus de perspectives différentes et proposent des interprétations divergentes, ce qui risque de produire des effets pervers. Elle peut en effet vider de leur sens politique les revendications féministes pour la reconnaissance du droit des femmes de décider en matière de reproduction; occulter les rapports sociaux qui façonnent les interventions de planification des naissances; et favoriser un glissement du discours féministe vers l'eugénisme. D'autres conséquences néfastes de cette " alliance tactique " sont liées à la représentation des femmes comme victimes de leur fécondité. En revanche, cette stratégie permet de faire face aux besoins urgents ressentis par de nombreuses femmes qui n'ont aucun accès à des services de planification des naissances.
Tous droits réservés @ Association des démographes du Québec, 1998

Ce document est protégé par la loi sur le droit d'auteur. L'utilisation des services d'Érudit (y compris la reproduction) est assujettie à sa politique d'utilisation que vous pouvez consulter en ligne.

https://apropos.erudit.org/fr/usagers/politique-dutilisation/ 
Cahiers québécois de démographie

Vol. 27, no 2, automne 1998, p. 253-265.

\title{
Discours féministe et néo-malthusianisme : les effets pervers d'une mésalliance
}

\author{
Maria DE KONINCK *
}

Le deux centième anniversaire de la publication de l'œuvre de Thomas R. Malthus, Essai sur le principe de population, constitue une occasion privilégiée pour réfléchir sur les débats et actions qui ont pris place depuis qu'il avait lancé la question de la pertinence d'un contrôle de la croissance démographique. Aborder la reproduction sous cet angle situe par ailleurs d'emblée au plan macro-social une réalité dont le fondement se trouve au plan micro-social, dans des expériences personnelles et interpersonnelles. D'autres approches sont possibles et permettent d'intégrer ces deux niveaux. Parmi elles, il y a la situation des femmes.

Les femmes ont depuis toujours, à ce que l'on sache (avec ou sans la connivence de leurs partenaires masculins), tenté de réguler les naissances par toutes sortes de méthodes préventives ou en recourant à l'avortement (Newman, 1985). Toutefois, ce n'est qu'à la fin du siècle dernier qu'a pris forme un discours de type militant associant cette régulation au statut des femmes, situant donc leurs expériences au plan politique; ce discours a été porté notamment par l'américaine Margaret Sanger.

La controverse qui entoure ce personnage historique parle d'elle-même. Elle tient au cheminement de Margaret Sanger, considérée par certaines comme une féministe (Chesler, 1992) mais accusée par d'autres d'avoir failli aux objectifs du mouvement et d'avoir adopté l'idéologie eugéniste (Hartmann, 1997; Hodgson et Watkins, 1997). Gloria Steinem apporte à son sujet la précision suivante. Selon elle, la motivation principale de

Université Laval, Département de médecine sociale et prëventive, Sainte-

Foy, Québec G1K 7P4. Courriel : maria.dekoninck@msp.ulaval.ca 
Sanger a toujours été l'amélioration de la condition des femmes; les alliances qu'elle a faites à un moment ou l'autre auraient été, en un sens, accessoires par rapport à cette préoccupation première dont l'origine, personnelle, était le décès de sa mère épuisée après 18 grossesses et 11 naissances vivantes (Steinem, 1998).

L'histoire des batailles menees par Sanger, peut-on prétendre, contenait déjà les paradoxes que l'on a retrouvés depuis dans des alliances et des confrontations entre les différents groupes préoccupés par la planification des naissances (DixonMueller, 1993), dont plusieurs s'articulent justement autour de ces deux niveaux de réalité. Encore aujourd'hui, les points de convergences entre le discours féministe et le néo-malthusianisme soulèvent la controverse.

Avant de poursuivre, il convient d'apporter quelques précisions sur les termes. Parler "du " discours féministe pourrait laisser entendre que ce discours est unique, alors qu'il est pluriel. Pour simplifier, dans le texte qui suit, nous emploierons toutefois le singulier, mais en désignant seulement certaines composantes des revendications formulees dans les lieux de rencontres internationaux au nom de l'amélioration de la condition des femmes et de leur droit de décider en matière de procréation. Décider de la procréation signifie choisir le moment, la fréquence, et enfin le contexte : entendons ici le partenaire et la nature du lien avec ce dernier. Le qualificatif de féministe se rapporte également ici à un mouvement politique et à son dénominateur commun, soit l'axe d'analyse des rapports sociaux de sexe. Son utilisation au singulier ne suppose toutefois pas l'existence d'un consensus au sein du mouvement féministe sur l'interprétation des différentes situations considérées.

Néo-malthusianisme désigne ici, par ailleurs, le mouvement inspiré de l'œuvre de Malthus qui préconise le contrôle de la croissance démographique au nom d'un mieux-être universel qui serait mis en danger par un déséquilibre éventuel (et réel dans certains milieux) entre les ressources et la population.

Ce texte vise à soulever un certain nombre de questions sur la controverse internationale entourant une alliance, sanctionnée lors de récentes conférences mondiales tenues sous l'égide des Nations Unies, entre des militantes porteuses d'un discours féministe et les néo-malthusiens (Simons, 1995), dans un contexte où, d'une part, s'exercent les pressions des groupes de femmes sur les politiques publiques (Dixon-Mueller et Germain, 1994), conjuguées à la présence de militantes féministes au 
sein d'organismes voués à la planification des naissances (Catley-Carlson, 1997; McIntosh et Finkle, 1995) et où, d'autre part, se diffuse une approche à visées globales recouverte par le terme "santé reproductive" (Lane, 1994; Faundes et Hardy, 1995). La controverse tient au fait que les objectifs poursuivis par chacun des deux mouvements ne semblent pas nécessairement compatibles alors que les deux discours se rejoignent dans les actions auxquelles ils donnent forme.

La thèse soutenue ici est que cette rencontre est en fait une mésalliance susceptible de générer des effets pervers pour la condition des femmes. L'argumentation qui sert à l'appuyer est la suivante : les préoccupations sous-jacentes à la construction des discours véhiculés par les deux mouvements découlent à la fois de perspectives et d'interprétations différentes sur la reproduction humaine et sur les enjeux en cause.

\section{DES PERSPECTIVES DIFFÉRENTES}

Il est clair en effet, soulignent Hodgson et Watkins (1997) après avoir analysé les alliances entre les deux mouvements, que leurs perspectives ne se situent pas au même niveau.

Bien qu'il soit un mouvement social et de revendications collectives, le mouvement féministe a articulé son discours sur la reproduction autour du sujet femme et de ses droits. S'il y a eu et s'il subsiste de nombreuses variantes sur la question, le droit des femmes de décider si elles vont devenir mères ou non est néanmoins un des fondements des luttes menées au nom du féminisme. Le slogan le plus caractéristique en cette matière est le suivant: "Nous aurons les enfants que nous voulons". Notons qu'historiquement le sens donné à cette volonté a plus souvent été celui de pouvoir refuser la procréation que l'inverse.

Or, la perspective néo-malthusienne a pour objet et unité de référence l'ensemble de la population. Même si elle peut s'intéresser à des situations circonscrites et locales et à de petites unités, son approche est celle des grands ensembles.

Cette différence de perspective est majeure. Elle est particulièrement signifiante parce que plusieurs études qui fondent les analyses féministes mettent l'accent sur la connaissance et la compréhension du quotidien et sur le rôle des acteurs dans la construction de la réalité sociale. Ces deux pôles, à l'échelle micro-sociale, constituent en effet un ancrage pour la lecture des expériences des femmes : de ce qu'elles vivent au quotidien 
et des réseaux de relations dans lesquels s'inscrivent leur vie de tous les jours (Smith, 1987).

Les deux démarches n'accordent pas le même statut aux droits individuels. Les féministes en font une fin, alors que les néo-malthusiens, comme le soulignent Hodgson et Watkins (1997), voient dans la reconnaissance des droits individuels un moyen indispensable pour atteindre des fins qui concernent un ensemble.

Lorsque les néo-malthusiens (au mieux) se rallient au discours féministe ou (au pire) en utilisent le contenu pour préconiser l'accès des femmes à la possibilité de planifier les naissances, ils le font sur d'autres bases que la reconnaissance de leurs droits. Il est capital de souligner que si " l'opérationnalisation" des objectifs néo-malthusiens appelle une prise en compte des rapports entre les sexes, celle-ci sera intégrée dans leurs actions, mais le but recherché n'est jamais de mettre fin aux inégalités entre les hommes et les femmes ni de changer les rapports entre les sexes. Il s'agit plutôt de favoriser des activités destinées à gérer la croissance de la population et allant, de façon secondaire, dans le sens des objectifs féministes.

À ces différences de perspectives s'ajoutent des divergences dans les interprétations des enjeux.

\section{DES INTERPRÉTATIONS DIVERGENTES}

L'interprétation inhérente au discours féministe sur la procréation vise les rapports sociaux entre femmes et hommes, rapports de pouvoir considérés comme générateurs d'inégalités dans les droits et dans leur exercice. Même si elles sont loin de toujours se rejoindre, on peut affirmer que les analyses féministes présentent inévitablement la procréation comme un des lieux stratégiques où se construisent les inégalités (Ginsburg et Rapp, 1995).

Le néo-malthusianisme propose, lui, une interprétation qui peut être qualifiée d'essentiellement économique "au sens large " : il s'intéresse au rapport de l'être humain à son environnement et aux ressources auxquelles il a accès. La procréation est située dans ce cadre, c'est-à-dire selon sa contribution à l'équilibre ou au déséquilibre du rapport.

Alain Drouard, dans son analyse des origines de l'eugénisme en France, cherche à montrer que celui-ci se développe "sur un terrain préparé par le néo-malthusianisme, avec lequel 
il va jusqu'à se confondre et qui, du fait de cette confusion, a souvent été occultê "(1992: 436). "Pour les néo-malthusiens comme pour les eugénistes, poursuit-il, question sexuelle et question sociale ne font qu'une seule et même question, à résoudre de manière "scientifique" sans intervention de l'État". Il y a effectivement des lieux de convergence entre néo-malthusianisme et eugénisme. Sur le fond, au-delà des apparences, il arrive que celui-ci trouve un écho auprès des néo-malthusiens, notamment lorsque la lecture économique juge certaines populations plus menaçantes que d'autres pour l'équilibre entre les êtres humains et l'environnement à l'échelle de la planète.

\section{LA RENCONTRE}

Malgré leurs différences, les interprétations néo-malthusiennes et féministes semblent parfois se rejoindre lorsqu'il s'agit de mettre en place des activités qui visent à répondre, d'une part, à une préoccupation internationale en matière de population et, d'autre part, à un souci d'offrir aux femmes des services leur permettant d'exercer ce qui est souvent désigné comme leur "autonomie reproductive " ou de leur donner le droit de décider en matière de reproduction. C'est en effet dans la mise en œuvre de leurs priorités respectives que les tenants de l'une et l'autre approche se retrouvent sur le mème terrain.

La Conférence mondiale sur la population et le développement tenue au Caire en 1994 a abouti à un certain consensus au plan international. Cet événement historique, précédé de multiples rencontres et de négociations ardues, a été l'occasion d'alliances stratégiques dont l'une des manifestations a été le silence des néo-malthusiens. Stanley Johnson, dans son livre sur cette conférence, qualifie de paradoxal le fait que l'on y ait si peu traité de population, l'attention ayant porté surtout sur un ensemble de conditions sociales. C'était, écrit-il, comme si discuter de visées démographiques était dépassé (1995: 175).

Accueillie comme un changement de paradigme par plus d'un auteur (Johnson, 1995; McIntosh et Finkle 1995), l'adoption au Caire d'une approche de la santé reproductive décrite comme "globale " peut mème laisser croire que le néomalthusianisme a disparu. On a été jusqu'à parler de "l'enterrement de Malthus "(Johnson, 1995).

Cette affirmation mérite d'être contestée. Les néo-malthusiens se sont plutôt rangés derrière les militantes féministes. La 
question ne demeure pas moins: celles-ci auraient-elles pu obtenir le plan d'action issu de la conférence sans l'appui de porteurs de visions néo-malthusiennes membres de différentes délégations ?

Situé dans une perspective historique, ce résultat est l'aboutissement de batailles menées dès la conférence de Bucarest, en 1974, par des féministes cherchant à assurer que les conditions de vie des femmes soient prises en considération dans les discussions internationales en matière de population. On peut faire le constat suivant: les féministes ont réussi à se faire entendre et les néo-malthusiens semblent maintenant comprendre que l'amélioration de la condition féminine est essentielle pour freiner la croissance démographique, notamment là où elle est la plus forte, dans les pays en voie de développement.

Une question accompagne ce constat. Cet appui peut-il nuire à l'avancement de la cause des femmes défini selon une perspective féministe, c'est-à-dire à la transformation des rapports sociaux de sexe ? Répondre à cette question serait réducteur puisque les dimensions à prendre en considération sont multiples, mais on peut formuler l'hypothèse que cette alliance est susceptible de générer au moins trois principaux effets pervers. Cette hypothèse sera êtayée dans la section suivante.

\section{LA PERTE DU SENS POLITIQUE}

Le premier effet pervers, sans doute le plus menaçant, serait que le projet inhérent au discours féministe en matière de reproduction soit vidé de son essence politique, à savoir la remise en cause des rapports sociaux. Le fondement politique du projet féministe, la redéfinition des rapports entre hommes et femmes, est loin de correspondre aux objectifs des actions prônées par les néo-malthusiens, soit le contrôle de la fécondité et non la transformation des rapports hommes-femmes.

La collusion entre des féministes et des néo-malthusiens, fût-elle circonstancielle, facilite notamment le glissement des constats et des interventions en matière de reproduction vers le champ exclusif de la santé, où la dimension politique des rapports sociaux peut ètre neutralisée. La santé, surtout s'il s'agit de la santé physique, est un domaine qui permet de gérer les situations sociales sans nécessairement s'attaquer à leurs fondements.

Par exemple, l'utilisation de l'argument du taux de mortalité maternelle (Morsy, 1995) pour justifier l'accès à la contracep- 
tion peut relever de la fausse représentation; on peut aussi intervenir comme s'il s'agissait d'une situation indêpendante des inégalités dans les rapports entre les sexes (De Koninck, 1998) '. Le cas des mutilations génitales féminines est sans doute l'exemple le plus probant des possibilités dont on dispose pour remettre des pratiques en cause au nom de la santé, sans révéler leur caractère profondément misogyne (IFD, s. d.).

De tels glissements sont possibles lorsqu'il s'agit d'assurer l'accès à la contraception et ils peuvent orienter non seulement les pratiques mais aussi la recherche dans le domaine de la contraception. Ainsi, dans plusieurs milieux, les femmes préfèrent utiliser des méthodes contraceptives qui leur permettent de ne pas informer leur partenaire. Engagées dans des relations où elles n'ont pas le droit d'exercer leur libre arbitre, elles le font quand même, souvent avec la complicité d'intervenant-e-s, et elles ont alors recours à des méthodes invisibles pour leur entourage. Cela serait l'une des raisons pour lesquelles certaines préfèrent les injections à la pilule contraceptive (Hardon, 1992).

Or, au point de vue de la santé des femmes, l'efficacité des méthodes n'est pas la seule préoccupation; leurs effets tels que les femmes les ressentent et l'importance qu'elles leur accordent comptent également. C'est pourquoi on note une différence dans l'évaluation des méthodes proposées selon qu'elle est faite par leurs usagères ou leurs promoteurs (Hardon, 1992).

Certes, situees dans une perspective à moyen terme, ces pratiques peuvent apparaitre comme une solution qui permet aux femmes de faire des choix et devrait contribuer à faire admettre par les collectivités où elles vivent leur droit de faire ces choix ouvertement. Il peut aussi y avoir des retombées positives pour ces femmes puisqu'elles arrivent ainsi à se donner un peu plus d'espace dans leur vie personnelle.

Néanmoins, on peut prétendre qu'une telle utilisation de la contraception peut avoir des effets sur la santé des femmes audelà de la seule dimension des grossesses non souhaitées, qu'elle accentue la distance entre les hommes et les femmes et qu'elle maintient le statut d'illégitimité de la planification des naissances, tout en évacuant totalement la responsabilité masculine.

1 Il ne s'agit pas ici de remettre en cause les programmes d'intervention en santé maternelle, mais plutôt d'attirer l'attention sur le fait que, dans certains contextes, ils peuvent servir à des fins autres que celles qui êtaient prévues; leur portée peut aussi être restreinte pour des raisons politiques. 
Ces situations interpellent particulièrement les personnes qui œuvrent auprès des femmes. Dans la poursuite d'un objectif de santé globale et selon une perspective coûts-bénéfices, la solution à court terme qui consiste à faciliter l'utilisation de la contraception rejoint le double objectif de contrôler les naissances et de permettre aux femmes de dêcider, même si elles le font secrètement. Mais elle rend aussi complice de l'absence de remise en cause des rapports sociaux générateurs d'inêgalités et de leur institutionnalisation.

\section{LA CONSOLIDATION DE RAPPORTS INÉGAUX}

Le second effet pervers possible de l'alliance des féministes avec les néo-malthusiens découle de la situation qui prévaut dans plusieurs milieux où les rapports sociaux sont empreints de hiérarchie. Les objectifs poursuivis par l'un et l'autre mouvement étant différents, les contextes dans lesquels les projets d'intervention en planification des naissances deviennent opérationnels ne font pas l'objet d'une analyse critique avant leur implantation. Cette lacune rend d'autant plus piégées les actions adoptées par les milieux de pratiques. En d'autres mots, on pense des programmes et on organise des services sans nécessairement tenir compte des rapports sociaux qui vont leur donner forme. Notamment, certains milieux très hiérarchisés favoriseront des actions de nature coercitive même si l'esprit des programmes suggérés ne va pas en ce sens (De Koninck, 1997).

Ainsi, dans plusieurs milieux où l'on entreprend des actions pour favoriser la régulation des naissances, les rapports de sexe influent sur l'accessibilité et la qualité des services. Les dimensions socioculturelles de l'utilisation de ces derniers, notamment celles qui ont trait aux rapports de sexe et aux relations entre les intervenant-e-s et les clientes, ne sont guère prises en considération. Par exemple, dans les sociétés où les rapports de classe sont marqués, les relations entre le personnel des services de santé et la clientèle reflètent les inégalités (Jaffre et Prual, 1994) et peuvent même les consolider (De Koninck, 1998).

Dans certains cas, ce sont les objectifs de population qui prévalent et colorent les services offerts aux femmes, dont la définition pourrait être différente s'ils visaient l'amélioration de la situation des utilisatrices. Ainsi, parfois, l'information est essentiellement orientée vers l'adoption de la contraception plutôt 
que vers le processus décisionnel (Sen et al., 1994), et l'évaluation des programmes porte sur le nombre de personnes qui. adoptent une méthode contraceptive et non pas sur la durée de leur recours aux services ni sur leur satisfaction.

Le discours féministe préconise, en matière de procréation, un soutien aux femmes dans leurs démarches pour arriver à faire des choix. Cette approche exige une mise au jour préalable des rapports sociaux dans lesquels s'inscriront les pratiques afin que leurs éventuels effets nocifs puissent être atténués.

La prise en charge de leur vie reproductive par les femmes ne rejoint toutefois pas nécessairement le modèle de gestion sociale de la reproduction inhérent au néo-malthusianisme. Ainsi, la préférence pour "l'espacement des naissances " plutôt que pour leur limitation manifestée par de nombreuses femmes dans différents milieux traduit peut-être une résistance à un modèle que l'on voudrait universel (Pelchat, 1995).

\section{L'EUGÉNISME}

Le glissement vers l'eugénisme serait le troisième effet pervers potentiel de l'alliance entre féministes et néo-malthusiens. Une crainte s'exprime au sein du mouvement des femmes eu égard à l'absence de limites dans le discours égalitaire en matière de procréation. Les glissements possibles concernent le droit de décider en matière de procréation et les normes sociales et éthiques qui devraient l'encadrer. Où s'arrête le droit de décider ? Ce droit justifie-t-il celui d'exiger la fabrication d'enfants en laboratoire et de choisir leurs caractéristiques sur la base de diagnostics prénatals? Ces questions de nature éthique sont également soulevées à partir des préoccupations néo-malthusiennes concernant la croissance de la population dans certaines sociétés et dans certaines couches sociales. En d'autres mots, en s'alliant avec les néo-malthusiens, les militantes féministes qui œuvrent dans le domaine de la reproduction se placent dans une situation où l'eugénisme peut progressivement trouver dans leur discours une certaine forme de légitimation.

L'inscription dans un cadre d'action appuyé par un mouvement dont la raison d'être est de freiner la croissance de la population pour favoriser l'épanouissement de la communauté humaine signifie aussi l'inscription dans une demarche qui :

- est ouverte à l'idée de la sélection (principe de base de l'eugénisme); 
- peut occulter les inégalités socio-économiques qui influent sur la disponibilité des ressources dont on prétexte la rareté pour limiter la croissance des populations; certains milieux sont en effet plus vulnérables que d'autres au déséquilibre parce qu'ils sont plus faibles au plan financier;

- instaure un biais dans la perspective dans la mesure où la reproduction humaine, que l'on veut contrôler, ne jouit pas du même poids que les préoccupations environnementales.

\section{CONCLUSION}

Au-delà de ces considérations et à titre de conclusion, une dernière dimension mérite d'être soulevée, celle des représentations des femmes et de la reproduction qui traversent les discours traitant du contrôle de la croissance démographique.

Ces représentations revêtent les formes suivantes. Les femmes se retrouvent au centre des discours mais elles n'y apparaissent pas nécessairement avec leurs qualités de personnes et d'actrices historiques et sociales. Elles y figurent comme les personnages clés de la croissance de la population, que l'on doit (selon les milieux) convaincre ou contraindre de gérer la reproduction pour éviter des problèmes sociaux, économiques et environnementaux.

Lorsqu'on leur prête une capacité de prendre des décisions, c'est dans un sens précis : il s'agit de décider pour contrôler ce qui, en demeurant incontrôlé, générera éventuellement le chaos. Parmi les multiples représentations des femmes, notons les suivantes : elles sont impuissantes, incarnent la vulnérabilité, sont des agentes de destruction de l'environnement et ainsi de suite.

Ces représentations livrent un message selon lequel les femmes sont victimes de leur fécondité et nuisent à l'équilibre environnemental. Une telle vision occulte les rapports sociaux de sexe générateurs d'inégalités. Lorsque le discours néomalthusien cède la place au discours féministe pour favoriser la mise en place de programmes de planification des naissances, c'est cette perception qu'il sanctionne et non pas celle de femmes vivant des inégalités sociales. La fécondité à contrôler, inscrite dans le corps des femmes, nous renvoie à une problématique de populations et de ressources. En revanche, la fécondité perçue comme une ressource humaine enjeu de rapports sociaux nous renvoie à ces rapports. 
Le contrôle de la fécondité en tant que préoccupation dominante se situe en opposition avec les objectifs mis de l'avant par les tenantes du discours féministe qui revendiquent dans les instances internationales le respect de la dignité des femmes en matière de reproduction et leur droit de prendre des décisions concernant leur vie reproductive.

Tenants du néo-malthusianisme et féministes réclamant en chœur la possibilité de mettre en place des services en matière de reproduction vont peut-être à court terme y trouver leur compte les uns et les autres. Mais à moyen et à long termes cette "mésalliance " risque d'avoir pour effet de consolider la représentation des femmes comme victimes de leur corps et de leur fécondité potentielle. Elle peut aussi contribuer à consolider la représentation de la reproduction (dont les femmes sont l'incarnation) comme un problème à gérer et non comme le fondement de la continuité humaine. Cette continuité pourrait pourtant, dans un contexte de rapports sociaux de sexe harmonieux, prétendre au titre de ressource la plus riche de l'humanité.

L'analyse critique présentée ici ne vise pas à juger les militantes féministes qui ont à un moment ou l'autre accepté de s'entendre avec des néo-malthusiens mais plutôt à attirer l'attention sur les conséquences néfastes qui peuvent découler de ces accords. En effet, il faut aussi reconnaitre que si les féministes n'ont pas dénoncé à hauts cris les visions néomalthusiennes de certains de leurs alliés stratégiques au cours des dernières années, c'est parce qu'elles sont animées par un sentiment d'urgence, urgence d'intervenir pour permettre à plus de femmes d'avoir accès à des modes de régulation des naissances qui, dans certains cas, leur procureront un peu de marge de manœuvre dans leur vie. Urgence aussi de trouver tous les moyens possibles pour tenter de faire diminuer le nombre de femmes qui meurent parce qu'elles sont enceintes trop souvent ou à de mauvais moments, ou parce que le refus de la grossesse les conduit à recourir à un avortement dans des conditions qui ne leur permettront pas d'y survivre.

Cette urgence devient alors sans doute pour les militantes féministes une raison suffisante pour courir le risque en espérant que les effets pervers de leurs alliances stratégiques seront contrés par d'autres interventions visant l'amélioration des conditions de vie des femmes. 


\section{RÉFÉRENCES BIBLIOGRAPHIQUES}

CATLEY-CARLSON, Margaret, 1997. "Implementing family planning programs in developing countries: Lessons and reflections from four decades of Population Council experience ", International Journal of Gynecology \& Obstetrics, 58, 1 : 101-106.

CHESLER, Ellen, 1992. Margaret Sanger: Woman of valor and the birth control movement in America. New York, Simon and Shuster.

DE KONINCK, Maria, 1997. "Le discours des femmes sur leur santé, un savoir essentiel pour l'intervention ", Recherches féministes, 10, $1: 97-112$.

DE KONINCK, Maria, 1998. "Reflections on the transfer of "progress": The case of reproduction", dans THE FEMINIST HEALTH CARE ETHICS RESEARCH NETWORK. The Politics of Women's Health. Exploring Agency and Autonomy. Philadelphie, Temple University Press : 150-177.

DIXON-MUELLER, Ruth, 1993. Population Policy \& Women's Rights. Transforming Reproductive Choice. Westport, Praeger.

DIXON-MUELLER, Ruth, et Adrienne GERMAIN, 1994. "Population policy and feminist political action in three developing countries", dans Jason L. FINKLE et C. Alison MCINTOSH, èd. The New Politics of Population. Conflicts and Consensus in Family Planning. Supplément, Population and Development Review, 20 : 197-219.

DROUARD, Alain, 1992. "Aux origines de l'eugénisme en France : le néo-malthusianisme (1896-1914) ", Population, 2 : 435-460.

FAUNDES, A., et E. HARDY, 1995. "From birth control to reproductive health ", International Journal of Gynecology \& Obstetrics, 49 : 55-62.

GINSBURG, Faye, et Rayna RAPP, ed., 1995. Conceiving the New World Order. The Global Politics of Reproduction. Berkeley, University of California Press.

HARDON, Anita Petra, 1992. "The needs of women versus the interests of family planning personnel, policy-makers and researchers: Conflicting views on safety and acceptability of contraceptives ", Social Science and Medicine, 35, $6: 753-766$.

HARTMANN, Betsy, 1997. "Population control 1: Birth of an ideology ", International Journal of Health Services, 27, 3 : 523-540.

HODGSON, Dennis, et Susan Cotts WATKINS, 1997. "Feminists and neo-Malthusians: Past and present alliances ", Population and Development Review 23, 3 : 469-523.

IFD (Institut de formation pour le développement), s. d. Les Conséquences de la circoncision féminine sur la santé. Cours de formation à l'intention du personnel médical, Cinquième module. Chapel Hill. 
JAFFRE, Yannick, et Alain PRUAL, 1994. "Midwives in Niger: An uncomfortable position between social behaviours and health care constraints ", Social Science and Medicine, 38, 8 : 1069-1073.

JOHNSON, Stanley, 1995. The Politics of Population. The International Conference on Population and Development, Cairo 1994. Londres, Earthscan Publications Ltd.

LANE, Sandra D., 1994. "From population control to reproductive health: An emerging policy agenda ", Social Science and Medicine, 39, $9: 1303-1314$.

MCINTOSH, C. Alison, et Jason L. FINKLE, 1995. "The Cairo Conference on Population and Development: A new paradigm?" Population and Development Review, 21, 2 : 223-260.

MORSY, Soheir, 1995. "Deadly reproduction among Egyptian women: Maternal mortality and the medicalization of population control ", dans Faye GINSBURG et Rayna RAPP, éd. Conceiving the New World Order. The Global Politics of Reproduction. Berkeley, University of California Press : 162-176.

NEWMAN, Lucile F., 1985. Women's Medicine. A Cross-Cultural Study of Indigenous Fertility Regulation. New Brunswick, Rutgers University Press.

PELCHAT, Yolande, 1995. "La Conférence internationale sur la population et le développement du Caire: un parti pris pour les femmes? ", Recherches féministes, 8, 1 : 155-163.

SEN, Gita, Adrienne GERMAIN et Lincoln C. CHEN, éd. 1994. Population Policies Reconsidered. Health, Empowerment, and Rights, Cambridge, Harvard Series on Population and International Health.

SIMONS, Helen, 1995. "Cairo: Repackaging population control ", International Journal of Health Services, 25, 3 : 559-566.

SMITH, Dorothy E., 1987. The Everyday World as Problematic. Toronto, University of Toronto Press.

STEINEM, Gloria, 1998. "Margaret Sanger ", Time Magazine, Canadian Edition, 151, 14 : 67-68. 